\title{
An Experience with Severe Leptospirosis (Weil's Disease): A Case Report
}

\author{
Jinnat Fatema Saira Safa ${ }^{1^{*}}$ \\ Farhana Mahmood ${ }^{2}$ \\ Sheikh Md Hasan Mamun \\ Mohammad Ferdous ${ }^{2}$ \\ Mohammad Jalaluddin ${ }^{3}$ \\ ${ }^{1}$ Department of Internal Medicine \\ Chattagram Maa-O-Shishu Hospital Medical College \\ Chittagong, Bangladesh. \\ ${ }^{2}$ Department of Internal Medicine, \\ National Hospital Ltd \\ Chittagong, Bangladesh. \\ ${ }^{3}$ Department of Community Medicine \\ Chattagram Maa-O-Shishu Hospital Medical College \\ Chittagong, Bangladesh.
}

*Correspondence to:

Dr. Jinnat Fatema Saira Safa

Assistant Professor

Department of Internal Medicine

Chattagram Maa-O-Shishu Hospital Medical College

Chittagong, Bangladesh.

Email: jinnatcmc@yahoo.com

Mobile: +8801534545252

www.banglajol.info/index.php/CMOSHMCJ

\begin{abstract}
Leptospirosis, a zoonotic disease is increasingly being recognized in developing countries. It is often underdiagnosed resulting in significant mortality as the presenting features mimic other commonly prevailing diseases like Malaria, Dengue, Acute hepatitis, Typhoid in developing world. We report a case of $32 \mathrm{yrs}$ old male, sewer worker presented with acute febrile illness with icterus, hemorrhagic manifestation and pulmonary-renal involvement, diagnosed as Severe Leptospirosis (Weil's disease) confirmed by ICT method. This experience highlights the importance of considering Leptospirosis early in the diagnosis of acute non specific febrile illness with multi-organ involvement to cutoff mortality from this fatal disease.
\end{abstract}

Key words: Leptospirosis; Zoonotic disease; Weil's disease; Multiorgan involvement; Fatal disease

\section{INTRODUCTION}

Leptospirosis is a zoonotic infection caused by spirochetes of the genus Leptospira. Infection usually results when water or soil contaminated with the urine of an infected animal comes in contact with human skin or mucous membranes. Clinical manifestations of leptospirosis can range from a self-limited flu like febrile illness to a fatal illness (Weil's disease) characterized by jaundice, hemorrhage, renal failure, severe pulmonary hemorrhage and $\mathrm{ARDS}^{1}$. In tropical settings, leptospirosis can be indistinguishable from other febrile illnesses such as malaria, dengue, viral hepatitis, other bacterial sepsis. Leptospirosis has been reported in neighboring areas of Southeast $\mathrm{Asia}^{2}$. But the disease is rarely recognized in Bangladesh where diagnostic tests for leptospirosis are less available. However, environmental factors, such as floods, humidity, and water contamination, are amenable to spread of the disease in Bangladesh. A study was conducted at 2 major hospitals in Dhaka, Bangladesh, during an outbreak of dengue fever. A total of $18 \%$ of dengue-negative patients tested positive for leptospirosis. The case-fatality rate among leptospirosis patients $(5 \%)$ was higher than among dengue fever patients $(1.2 \%)^{3}$. This case report will update the reader about leptospirosis, a possible diagnosis in febrile illness with multiorgan involvement.

\section{CASE REPORT}

A previously healthy 32 yrs old male admitted into Chattagram Maa-O-Shishu Hospital (CMOSH) with the complaints of high grade fever, anorexia, generalized body ache for 2 days. He had occasional dry cough and no other symptoms. He gave no history of recent travelling, blood transfusion, contact with sick person and significant past illness. 
He was nonsmoker, nonalcoholic, sewer worker at CMOSH and worked in flooded area during rainy season. Physical examination revealed high temperature $\left(102^{\circ} \mathrm{F}\right)$ conjunctival congestion, pulse rate $84 / \mathrm{min}$, blood pressure $120 / 80 \mathrm{~mm}$ of $\mathrm{Hg}$, respiratory rate $28 / \mathrm{min}$, systemic examinations were unremarkable. He was diagnosed initially as a case of Flu and discharged after 2 days as became afebrile. But he had persistant malaise and within a day or two he developed upper abdominal pain, vomiting, yellow colouration of eyes and scanty high coloured urine. He had diarrhea for episodes of 2-3 and started G-I bleeding in the form of hematemesis and malena. He also noticed haemoptysis once. At readmission, he was found conscious, well alert, deeply icteric with conjunctival suffusion, respiratory rate $>46 / \mathrm{min}$, pulse rate 120 beats $/ \mathrm{min}$, blood pressure $100 / 60 \mathrm{~mm}$ of $\mathrm{Hg}$, tem $101^{\circ} \mathrm{F}$, bilateral lung base crepitations, tender epigastrium and hepatomegaly . Acute viral hepatitis, Malaria, Dengue, Sepsis with mutiorgan failure and Leptospirosis were in the list of differentials. Investigations with results are shown in the following tables:

Table 1 : Laboratory Data.

\begin{tabular}{|c|c|c|c|}
\hline Serum Chemistry & 1st Sample & Subsequent sample & Reference value \\
\hline Sodium (mmol/L) & 134.0 & 144.2 & 136-145 \\
\hline Potassium (mmol/L) & 3.7 & 3.4 & $3.5-5$ \\
\hline Chloride (mmol/L) & 961 & 11.0 & $96-106$ \\
\hline Bicarbonate $(\mathrm{mmol} / \mathrm{L})$ & 26.7 & 22.4 & $25-30$ \\
\hline Creatinine (mg/dl) & 5.8 & 6.4 & $0.7-1.3$ \\
\hline Urea (mg/dl) & 170 & 781 & $5-45$ \\
\hline S. Bilirubin total (mg/dl) & 25.4 & 44.2 & $<1.10$ \\
\hline Direct. Bilirubin (mg/dl) & 20.0 & 32.0 & \\
\hline Indirect bilirubin (mg/dl) & 5.4 & 12.2 & \\
\hline Alkaline phosphatase & & $\mathrm{U} / \mathrm{L}$ & 40-190U/L \\
\hline SGPT (ALT) (U/L) & 74 & 57 & $10-40 \mathrm{U} / \mathrm{L}$ \\
\hline SGOT (AST) (U/L) & 38 & 39 & 15-30U/L \\
\hline Hematology & 1st Sample & Subsequent sample & Reference value \\
\hline $\mathrm{Hb}$ & 14.6 & 11.2 & M13.5-17.5,F12-16 \\
\hline WBC & 9,000 & 16,600 & $4000-11000$ \\
\hline PLT & $2,60,000$ & $1,50,000$ & $1,50,000-400000$ \\
\hline ESR & 30 & 90 & M0-15,F0-20 \\
\hline Coagulation profile & 1st Sample & Subsequent sample & Reference value \\
\hline PT & 14 & 16 & P14 sec,C14sec \\
\hline INR & 1.0 & 1.18 & 1 \\
\hline aPTT & 34 & & $26-36$ \\
\hline Urine $\mathrm{R} / \mathrm{E}$ & 1st Sample & Subsequent sample & Reference value \\
\hline $\mathrm{pH}$ & 8.5 & 7.0 & 5.5 to 7 \\
\hline S. gravity & 1010 & 1015 & 1010 to 1020 \\
\hline Albumin & ++ & Trace & nil \\
\hline $\mathrm{RBC}$ & Plenty & Plenty & nil \\
\hline Puscell & $7-8$ & $1-2$ & nil \\
\hline $\mathrm{ABG}$ & 1st Sample & Subsequent sample & Reference value \\
\hline $\mathrm{pH}$ & 7.40 & 7.39 & $7.35-7.45$ \\
\hline $\mathrm{PCO}_{2}$ & 27.8 & 39.2 & $35-45$ \\
\hline $\mathrm{PO}_{2}$ & 87.5 & 100.7 & $75-100$ \\
\hline $\mathrm{HCO}_{3}-$ & 16.9 & 22.4 & $22-28$ \\
\hline
\end{tabular}

Table 2 : Microbiological investigation.

\begin{tabular}{ll} 
Serological Test & Interpretation \\
Anti-HAV IgM & Negative \\
HbsAg & Negative \\
Anti-HCV & Negative \\
Anti-HEV IgM & Negative \\
NS1 antigen for Dengue & Negative \\
ICT for Malaria & Negative \\
Triple antigen/Fibrile antigen & Negative \\
IgM antileptospiral antibody (ICT) & Strongly positive \\
Blood culture & No growth after $72 \mathrm{hrs}$ \\
\hline
\end{tabular}

On Chest X- Ray P/A view there was bilateral diffuse patchy opacity and sinus tachycardia on ECG, Echocardiography was Normal, USG of abdomen revealed hepatomegaly with findings in favour of Acute hepatitis, both kidneys were swollen with ill defined corticomedullary differentiation suggestive of renal parenchymal disease .

The patient was managed in ICU with oxygen inhalation, maintenance of nutrition, hydration with I/V fluid, intravenous Ceftriaxone $1 \mathrm{gm} 12 \mathrm{hrly}$, I/V antiemetic, I/V proton pump inhibitor with strict monitoring. The patient had hemodialysis once for raising creatinine. On subsequent days creatinine had fallen gradually to normal level and output increased satisfactorily. Serum bilirubin had fallen slowly and patient had significant clinical recovery with normalization of hematological and biochemical parameter over a period of 3 wks.

\section{DISCUSSION}

Leptospirosis, has recently been recognized as a re-emerging infectious disease among animals and humans that has the potential to become more prevalent with anticipated global warming ${ }^{4,5}$. Leptospirosis peaks during the monsoon and postmonsoon months and occurs more commonly where poor sanitation and low hygienic conditions are prevalent ${ }^{6}$.

Transmission of leptospiral infections results from direct or indirect exposure to the urine of infected animals( most commonly rat). The natural course of leptospirosis comprises of two distinct clinical phases: septicemia and immune phase. Seven to twelve days post exposure patient developes nonspecific flu-like illness with sudden onset of high fever, headache, myalgia and conjunctival suffusion (Septicaemia phase $)^{7}$. While body's immunologic response leads to production of immunoglobulin $\mathrm{M}$ antibodies and specific organ damage can be observed ( Immune phase) ${ }^{7}$. During this phase, aseptic meningitis, Acute Renal Injury (AKI) pulmonary hemorrhage and Acute Respiratory Distress Syndrome (ARDS) may occur. This most severe form of leptospirosis is the Weil's disease ${ }^{7}$. 
Our patient presented with leptospirosis in its severe form, i.e. icteric-hemorrhagic illness with multiorgan dysfunction (Weil's disease). Initially he had non specific symptoms of Flu like illness, later he developed symptoms of multiorgan involvement (Jaundice, G-I bleeding, hemoptysis, oliguria) which let us think about the rare but fatal disease leptospirosis. The major cause of death in patients with Weil's disease is severe pulmonary hemorrhagic syndrome ${ }^{8}$.Our patient had pulmonary involvement in the form of cough and hemoptysis along with the radiological evidence of diffuse, ill-defined ground glass density in both lung field, that has been described in leptospirosis ${ }^{8}$. This radiologic abnormality was suggestive of alveolar hemorrhage because of faster resolution (Within a week) in comparison to other forms of bacterial pneumonia which resolve slowly.

Hepatic dysfunction is usually not severe in leptospirosis and reversible. But in severe leptospirosis, liver dysfunction can be seen as conjugated serum bilirubin levels may increase to above $80 \mathrm{mg} / \mathrm{dl}$, accompanied by moderate elevations in transaminases, which rarely exceed $200 \mathrm{U} / \mathrm{L}^{9}$. Conjugated hyperbilirubinaemia and mild elevation of SGPT but normal Alkaline phosphatase were evident in this case aswell.

Renal impairment is frequently seen in Weil's disease. Azotemia, oliguria, and anuria commonly occur during the second week of the illness but may apear as early as 3 to 4 days after the onset ${ }^{10}$. In this case there was hematuria, pyuria, protinuria and AKI. Serum creatinine was raised upto 6.4 $\mathrm{mg} / \mathrm{dl}$ and following single episode of dialysis gradually became normal.

The gold standard for diagnosis of leptospirosis is isolation of the organism by culture of clinical specimens (Blood, CSF, urine) during the first seven to 10 days of the illness. However, this method requires special media and more than 16 weeks because initial growth may be slow and has a low sensitivity and specificity. The majority of leptospirosis cases are diagnosed by serological method, MAT (Microscopic Agglutination Test). MAT is unavailable in our laboratory. Here diagnosis was done by IgM anti leptospiral antibody detection with ICT method. ICT test is moderately sensitive but highly specific ${ }^{11}$.

The current treatment of choice for mild leptospirosis includes oral doxycycline and amoxicillin. In cases of fulminant leptospirosis parenteral high-dose penicillin $G$ has long been considered as the treatment of choice. Recent clinical trials have proved the acceptability of third generation cephalosporins: cefotaxime and ceftriaxone agents for patients with severe leptospirosis ${ }^{12}$. We empirically started inj ceftriaxon initially and later on added tab doxycycline. The patient responded with satisfactory clinical improvement.

Overall, mortality rate of Weil's disease is $5 \%$ to $10 \%$. The mortality is caused mainly by renal failure, cardiopulmonary failure and widespread hemorrhage ${ }^{13}$. So this fatal disease needs early notification, diagnosis and prompt initiation of specific antimicrobial.

\section{CONCLUSION}

Diagnosis of Leptospirosis is often a challenge as the presentations mimic other common diseases. A high index of suspicion is crucial if a patient presents with acute febrile illness with hepatic and renal dysfunction and prompt antibiotic therapy should be initiated on the basis of clinical judgement as laboratory confirmation can be delayed. Avilability of a quick, reliable diagnostic method (ICT test) should be ensured at local hospital for early diagnosis.

\section{DISCLOSURE}

All the authors declared no competing interest. 
REFERENCES

1. Levett PN Leptospirosis. Clin Microbiology, Rev. 2001;14:296-326.

2. Chaudhry R, Premlatha MM, Mohanty S, Dhawan B, Singh KK, Dey AB. Emerging leptospirosis, north India. Emerg Infect Dis. 2002;8:1526-1527.

3. Regina C, LaRocue, Robert F, Breiman, Mary D. Ari, Roger E.Morey, Firdous Ara Jahan, John Mosely Hayes, M. Anower Hossain, W. Abdullah Brooks and Paul N.Levett. Leptospirosis during Dengue Outbreak, Bangladesh; emerging infectious disease. 2005; 11(5):766-769.

4. Aruna Giri. An Indian experience with Leptospirosis, Home page, Available from http://drgiri.com/node/22 (Accessed on 20 December 2011).

5. Langston CE, Heuter KJ. "Leptospirosis. A re-emerging zoonotic disease". Veterinary Clinics of North America, Small Animal Practice. 2003:33(4):791-807.

6. Deodhar D, John M. Leptospirosis: Experience at a tertiary care hospital in northern India. The National Medical Journal of India. 2011:24(2):78-80

7. Levett PN, Haake DA. Leptospira species (Leptospirosis). In: Mandell GL, Bennett JE, Dolin R, editors. Principles and Practice of Infectious Diseases, Philadelphia: Churchill Livingstone Elsevier. 2010;3059-3065.

8. Vijayachari P, Sehgal SC, Goris MG, Terpstra WJ, Hartskeerl RA: Leptospira interrogans serovar Valbuzzi: A cause of severe pulmonary haemorrhages in the Andaman Islands. Journal of Medical Microbiology. 2003; 52(10):913-918.

9. Chang ML, Yang CW, Chen JC, Ho YP, Pan MJ, Lin CH, Lin DY. Disproportional exaggerated aspartate transaminase is a useful prognostic parameter in late leptospirosis. World Journal of Gastroenterology. 2005;11(35):5553-5556.

10. Farr RW: Leptospirosis. Clin Infect Dis. 1995;21:1-6.

11. Bajani MD, Ashford DA, Bragg SL, et al. Evaluation of four commercially available rapid serologic tests for diagnosis of leptospirosis. J Clin Microbiol. 2003; 41:803-809

12. Raptis L, Pappas G, Akritidis N: Use of ceftriaxone in patients with severe leptospirosis. International Journal of Antimicrobial Agents. 2006;28(3):259-261.

13. Terpstra W: Human Leptospirosis: Guidance for Diagnosis, Surveillance, and Control Geneva: World Health Organization. 2003. 